\title{
Energetic electrons in the magnetosphere of Saturn
}

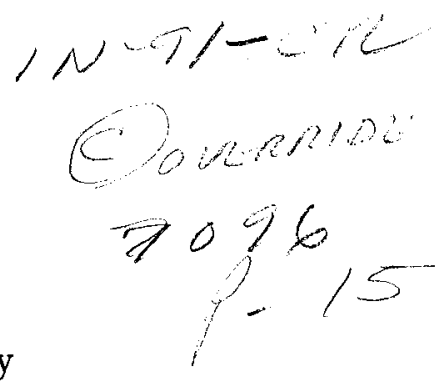

B. A. Randall

Department of Physics and Astronomy, University of Iowa, Iowa City

\begin{abstract}
The energy spectra and angular distributions of electrons observed by Pioneer 11 as a function of radial distance in the inner magnetosphere of Saturn are reanalyzed and phase space densities are then calculated. The radial dependence of phase space density requires a distributed loss process. The loss is greatest in the region of the $E$ ring $(5.5<L<8.5)$ and is attributed to collisions with the ring particles in agreement with earlier work by Van Allen et al. (1980b). Quantitative analysis yields the following properties of the $E$ ring: the particle radii are in the range of $4 \times 10^{-5}$ to $3.2 \times 10^{-4} \mathrm{~cm}$ and the thickness of the ring is approximately $3 R_{s}$. Between the inner edge of the $E$ ring $\left(5.5 R_{s}\right)$ and the outer edge of the $A$ ring $\left(2.3 R_{s}\right)$ there are more energetic electrons than can be supplied by radial diffusion from an external source. Detailed calculations show that a cosmic ray albedo neutron decay (CRAND) source in the $A$ and $B$ rings is a plausible source for this excess. The radial diffusion coefficient required to explain the $E$ ring absorption and CRAND source for electrons is $1 \times 10^{-12}>D_{0}>3 \times 10^{-12} R_{s}^{2} / \mathrm{s}$, assuming that $D_{L L}=D_{0} L^{3}$. As part of the reanalysis program, a method for the deconvolution of pitch angle distributions observed by simple detectors on a rotating spacecraft is developed. This process removes the instrumental response and rotational smear due to finite sampling periods and yields true angular distributions.
\end{abstract}

\section{Introduction}

The discovery and survey of the magnetosphere of Saturn by Pioneer 11 in 1979 showed that it is intermediate in size and particle population between those of the Earth and Jupiter. The interaction of the trapped particle population with the satellites and rings of Saturn provides a valuable basis for discussing the dynamics of a relatively quiescent magnetosphere. In one of the early papers on the Saturnian magnetosphere [ Van Allen et al., 1980b], the angular distributions, energy spectra, and radial distribution of the intensity of energetic electrons were shown to have distinctive features, and it was apparent that more definitive information could be obtained from this data set.

As part of the reanalysis of the data, raw angular distributions have been deconvolved to remove instrumental response and rotational smear. Twenty-eight samples at approximately every $0.5 R_{s}$ inside $9.5 R_{s}$ $\left(1 R_{s}=60,000 \mathrm{~km}\right)$ on both the inbound and outbound legs of the encounter trajectory were deconvolved. The deconvolved pitch angle distributions together with approximate energy spectra are used to calculate a family of improved phase space densities as a function of radial distance. The phase space densities are then used to study the radial diffusion of the energetic electrons

Copyright 1994 by the American Geophysical Union.

Paper number 94JA00254.

0148-0227/94/94JA-00254\$05.00 and to determine something about their sources and losses.

Preliminary phase space densities based on omnidirectional averages of these data were constructed [Van Allen et al., 1980b; Van Allen, 1984], but these assumed that the second adiabatic invariant was zero. Armstrong et al. [1983] calculated electron phase space densities from the Voyager data at Saturn, but their observational data did not extend to the outer edge of the $A$ ring and were limited to high latitudes.

\section{Deconvolution of Observed Angular Distributions}

The University of Iowa Geiger Tube Telescope (GTT) on Pioneer 11 [Van Allen et al., 1980b] has three directional detectors, designated $\mathrm{A}, \mathrm{B}$, and $\mathrm{G}$. The axes of their conical collimators are parallel to each other and orthogonal to the rotational axis of the spacecraft. The detectors are sampled at a rate that is not synchronous with the rotational rate. Also, it is noted that the rotational axis of the spacecraft is approximately orthogonal to the magnetic vector during Pioneer 11's nearequatorial encounter trajectory. Hence fairly complete angular distributions of intensities can be assembled in 231-s blocks of data. The direction of the local magnetic vector is determined by the onboard magnetometer [Smith et al., 1980]. The pitch angle of the axis of the detector at the midtime of each sample can be calculated, and raw angular distributions of particle intensities can be assembled. These observed distributions are 
distortions of the true ones because of the finite opening angle of the detector's collimator and the smear in rotational angle due to the finite sampling intervals. Van Allen and Grosskreutz [1989] adopted a convolutional approach to this problem by assuming a variety of true angular distributions of simple form and choosing the best one by trial and error. An improved approach is taken in this paper. As in previous work, it is assumed that the particle distributions are rotationally symmetric about the magnetic vector and that they have mirror symmetry with respect to a plane perpendicular to the magnetic vector, that is, there is no streaming of particles. This is the case for observations inside $L=10 R_{s}$. In the outer magnetosphere $\left(L>10 R_{s}\right)$, asymmetric angular distributions were seen by Pioneer 11 and both Voyagers. The true pitch angle distribution of the unidirectional integral intensities $J$ is represented by a finite series of the form

$$
J(\alpha)=J_{0}+\sum_{n=1}^{m} J_{2 n} \cos ^{2 n}(\alpha) .
$$

The pitch angle $\alpha$ of a particular line within the conical field of the collimator is related to the pitch angle of the axis of the collimator $\beta$ and the $\theta$ and $\phi$ coordinates of the chosen line by

$$
\cos \alpha=\cos \beta \cos \theta+\sin \beta \sin \theta \cos \phi .
$$

This relationship is shown in Figure 1a. The response (counting rate) of the detector is given by

$$
R(\alpha)=(\text { detector area }) \int_{0}^{2 \pi} d \phi \int_{0}^{\theta_{0}} \sin \theta d \theta F(\theta) J(\alpha)
$$

where $F(\theta)$ is the measured response of the detector and $\theta_{0}$ is the angle at which $F(\theta)=0$. Relations (1) and (2) are substituted into equation (3), and the integrals are evaluated term by term. The result is $R(\alpha)$ in terms of a power series in $\cos ^{2} \beta$.

The rotational smear due to the finite sampling time must next be taken into account. The general relationship between the detector, magnetic field, and the spacecraft spin axis necessary for this calculation is shown in Figure 1b. The details of this procedure and the final explicit formulae of the deconvolution process are given in the appendix.

Figure 2 shows an example of the data at $4.5 R_{s}$ on the inbound pass for detector A. This example is typical of most of the pitch angle distributions that have been analyzed between 3.5 and $10 R_{s}$. The background counting rate of detector $\mathrm{C}$ is only a few counts per second; hence no correction of the rate of detector $A$ is necessary. The solid curve is the least squares fit to the raw data using three terms (i.e., $B_{0}+B_{2} \cos ^{2} \beta+B_{4} \cos ^{4} \beta$ ). The dashed curve represents the deconvolved or "true" pitch angle distribution. This example shows the general effect of deconvolution, that is, the intensity is increased at large pitch angles and decreased at small pitch angles.

A second example is shown in Figure 3. This distribution is for detector $\mathrm{A}$ at $2.8 R_{s}$ on the inbound pass.
The background counting rate of detector $\mathrm{C}$ is shown by the solid curve at the bottom of the figure. The average rate of $\mathrm{C}$ is 1075 counts/s and there is a roll modulation amplitude of 167 counts/s for this nearly isotropic background detector. For this case the aver-


Figure 1. (a) The instantaneous angular relationship of the axis of the conical collimator and a particular line within the field of view, where $\beta$ is the angle between the magnetic field vector and the axis of the detector, $\alpha$ is the angle between the magnetic field vector and the chosen line, $\theta$ is the angle between the line and the axis of the detector, and $\phi$ is the azimuth of the line. The Cartesian coordinates are chosen such that the $Z^{\prime}$ axis is coincident with the detector axis and the magnetic field vector is in the $X^{\prime}-Z^{\prime}$ plane. (b) The instantaneous angular relationship between the axis of the detector, the vector magnetic field direction, and the spin axis of the spacecraft. The spin axis is taken to be along the $Z$ axis, $\Gamma$ is the colatitude of the magnetic field vector with respect to the spin axis, $\chi$ is its longitude as measured from an inertially fixed $X$ axis, $\lambda$ is the colatitude of the axis of the detector with respect to the spin axis, and $\eta$ is its longitude as measured from the $X$ axis. 


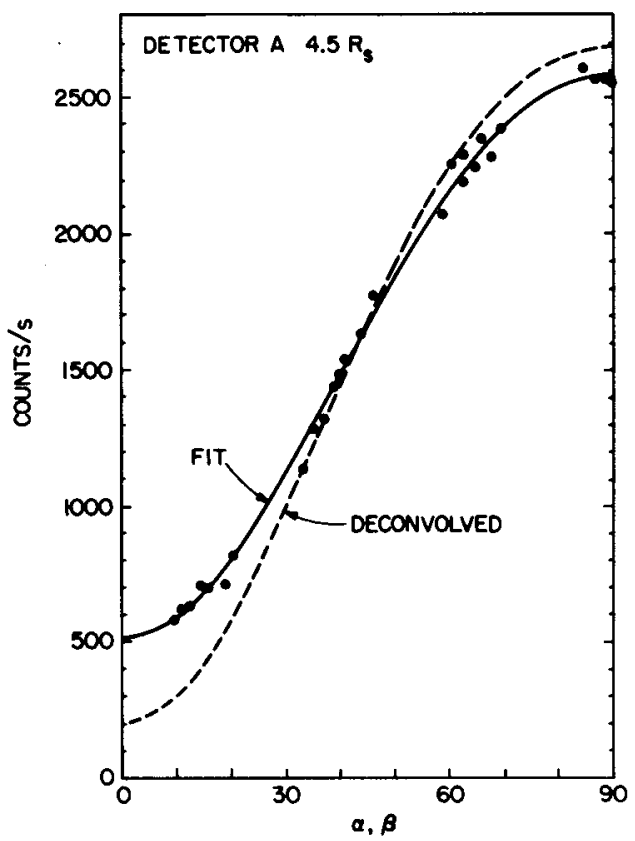

Figure 2. Pitch angle distribution of electrons. The plotted points are the observed data as a function of $\beta$. The solid curve is a three-term polynomial fit in $\cos ^{2} \beta$ to the data. The dashed curve shows the deconvolved pitch angle distribution as a function of $\alpha$.

age computed loss cone is $9.4^{\circ}$. The combination of the finite opening angle of the detector, the smear due to the finite sampling period over this large loss cone, and the depletion at $90^{\circ}$ makes the fitting of the data to a three-term series impossible. In this case, the data were least squares fit for pitch angles only greater than $25^{\circ}$ to avoid the effects of the loss cone. The data of principal interest for the purposes of this paper are for pitch angles greater than $60^{\circ}$. More careful fitting in the loss cone region should be done if this is the region of interest. The solid curve is the result of a four-term fit to the raw data; it shows a peak at $71.5^{\circ}$ and a decrease in intensity near $90^{\circ}$. A three-term fit to the same data shows no decrease near $90^{\circ}$. The deconvolved curve is shown as a dashed line. All of the pitch angle distributions inside $3.5 R_{s}$ that were studied showed depletion of intensities near $90^{\circ}$ and required the more detailed analysis to complete the deconvolution process. This depletion is indicative of losses in a ring of particulate material at the equator in this region [Thomsen and Van Allen, 1979]. The counting rates of detectors A and $\mathrm{B}$, after exiting from under the $A$ ring on the outbound leg of the trajectory, were substantially less than at the same radial distances on the inbound leg. Deconvolution showed that the pitch angle distributions were similar in shape but not in intensity. In contrast, detector $\mathrm{C}$, which was responding predominately to protons with energy greater than $80 \mathrm{MeV}$, measured nearly the same intensities on both legs. This effect on the electrons is evident in the data from the other energetic particle experiments on Pioneer 11 [Fillius et al., 1980]. The difference between the electron measure- ments inbound and outbound slowly became negligible with increasing radial distance. This matter has been discussed by Van Allen and Grosskreutz [1989].

\section{Scheme of Analysis}

In order to get an understanding of the dynamics of the magnetosphere of Saturn, the phase space densities as a function of radial distance, or magnetic shell parameter $L$, must be calculated. For Pioneer 11's nearequatorial encounter trajectory in Saturn's centered nontilted magnetic field, the value of $L$ in the inner magnetosphere is approximately equal to the radial distance in units of the planet's radius $\left(1 R_{s}=60,000 \mathrm{~km}\right)$. The phase space density $f$ is defined as $f=j /(p c)^{2}$, where $j$ is the differential intensity and $p c$ is the momentum. The values of $j$ are calculated at constant $\mu$ and $J$, the first two adiabatic invariants:

$$
\begin{gathered}
\mu \equiv \frac{p^{2} c^{2} \sin ^{2} \alpha_{0}}{2 m_{e} c^{2} B_{0}}, \\
J \equiv \oint p c \cos \alpha_{0} d s=2 p c L R_{s} I\left(\sin \alpha_{0}\right),
\end{gathered}
$$

where $B_{0}$ and $\alpha_{0}$ are the equatorial magnetic field strength and pitch angle, respectively, $m_{e}$ is the electron

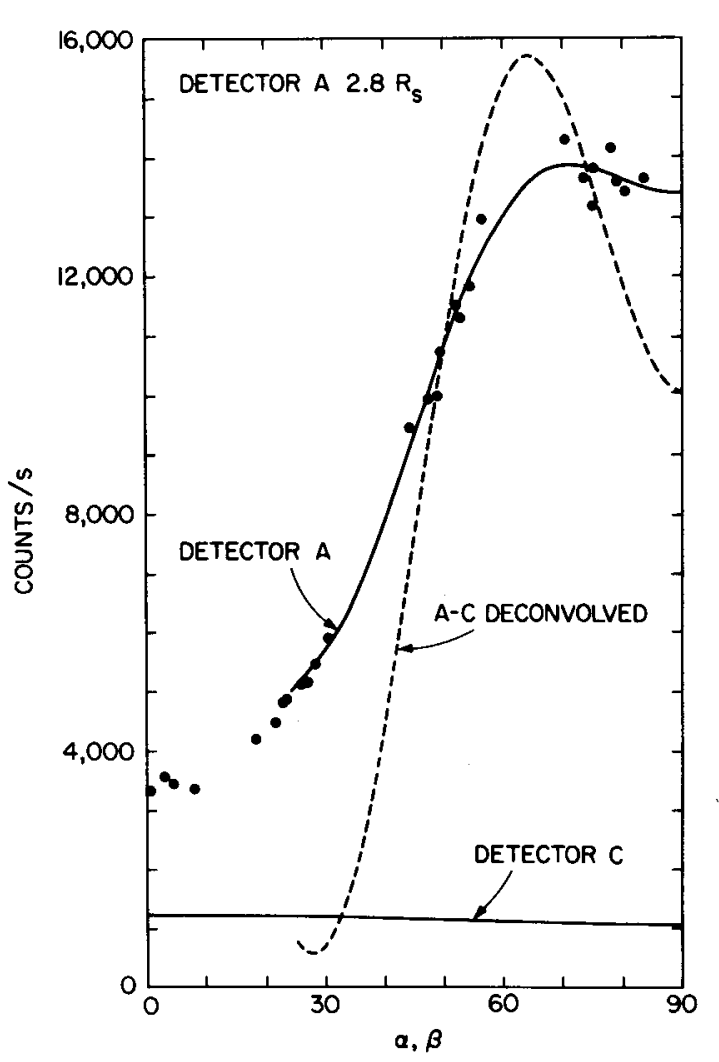

Figure 3. Similar to Figure 2. The solid curve, labeled detector A, is a four-term fit to the data for pitch angles greater than $25^{\circ}$. The lower solid curve is a two-term fit to the omnidirectional detector $\mathrm{C}$. The dashed curve, labeled $\mathrm{A}-\mathrm{C}$ deconvolved, is the inferred true angular distribution (see text). 
mass, $s$ is the curvilinear path length along the magnetic field, $y=\sin \alpha_{0}, I(y) \approx 2 U(1-y)+2 V(y \ln y+$ $2(y-\sqrt{y}))$, and $U=1+\ln (2+\sqrt{3}) / \sqrt{12}$, and $V=$ $U / 2-\pi / \sqrt{72}$ [Schulz, 1971].

The momentum $p c$ can be eliminated from the adiabatic invariants [Schulz and Lanzerotti, 1974] by taking the ratio

$$
\frac{J}{\sqrt{\mu}}=\sqrt{8 m_{e} c^{2} B_{0}} L R_{s} \frac{I\left(\sin \alpha_{0}\right)}{\sin \alpha_{0}}=K .
$$

Three values of $K$ were chosen such that the range of pitch angles sampled would be around $80^{\circ}, 70^{\circ}$, and $60^{\circ}$. These values in units of $G^{1 / 2} R_{s}$ are

$$
\begin{aligned}
& K_{1}=5.180 \times 10^{-3} \\
& K_{2}=1.951 \times 10^{-2} \\
& K_{3}=5.166 \times 10^{-2} .
\end{aligned}
$$

The McIlwain $L$ parameter was calculated at the midpoint position where each pitch angle distribution was assembled, from the $P_{11} 84$ model of Davis and Smith [1986]. The magnetic equator for each $L$ was determined using the model, and the corresponding minimum field strength was scaled from the measured field strength using the model. The corresponding pitch angles were calculated for each distribution, and the integral electron intensities were calculated for the deconvolved pitch angle distributions for both detectors A and $\mathrm{B}$. The integral spectra of electrons with kinetic energy $T>40 \mathrm{keV}$ (detector A) and $T>0.56 \mathrm{MeV}$ (detector B) correspond to a given value of $K$. To calculate the phase space density, the differential energy spectrum must be estimated for each radial distance.

In the region for $L>7.5$, the spectrum can be represented by $j(T)=k T^{-\gamma}[M c D o n a l d$ et al., 1980; Van Allen et al., 1980b]. At smaller radial distances, the intensity from detector B continues to increase with decreasing distance as if it were responding to a similar spectrum that was adiabatically transformed to smaller distances. The intensity from detector $\mathbf{A}$, on the other hand, decreases in intensity between $L=7.5$ and 5.5 and then starts to increase with an intensity slightly greater than that of detector $\mathrm{B}$. This can be seen in Figure 4. A two point determination of the spectrum can still be made in the region between $L=7.5$ and 5.5 , but the value of $\gamma$ must change drastically to account for the decrease of intensity in detector A relative to detector B. Nonrelativistically, the value of $\gamma$ does not change for a power law spectrum undergoing radial diffusion with constant $\mu$ and $J$. But the electrons detected by detectors A and B are mildly relativistic, so $\gamma$ is not a constant. To see how much $\gamma$ varies for relativistic electrons, the correct relativistic expressions must be used. The momentum is given by $p c=\left(T\left(T+2 m_{e} c^{2}\right)\right)^{1 / 2}$. Five values of $\gamma$ were determined for each $K_{i}$ at $L$ values of $7.5,8.0,8.5,9.0$, and 9.5 for the inbound data. Six differential spectral intensities were calculated between $40 \mathrm{keV}$ and $2 \mathrm{MeV}$, and these were converted to phase space densities. It was assumed that the phase space densities could be translated to $L=9$ by a loss-

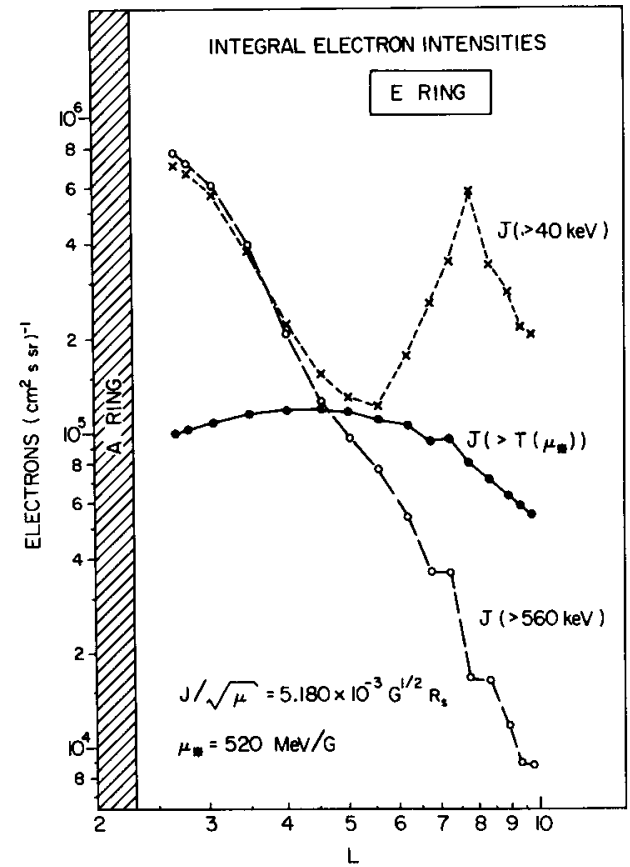

Figure 4. The integral electron energy intensity determined from the deconvolved pitch angle distributions of detectors A and B on Pioneer 11. These detectors have thresholds of 40 and $560 \mathrm{keV}$, respectively. The integral intensities are at a constant $J / \mu^{1 / 2}=$ $5.180 \times 10^{-3} G^{1 / 2} R_{s}$ and are plotted versus $L$. Note that the lower energy integral intensity decreases in the region denoted as the $E$ ring. The solid line with the solid circles represents the response of a detector with a threshold energy corresponding to a constant first adiabatic invariant $\mu_{*}$.

free diffusion process and would differ only by a factor that is a function of distance and is assumed to be independent of energy. The five differential spectra were recalculated using the values of $B_{0}$ and $\alpha_{0}$ to determine $T$ from the value of $\mu$. These five spectra were each least squares fit to find the transformed value of $\gamma$ at $L=9$. The average value of $\gamma$ was determined from these five values for each $K_{i}$. This is equivalent in a sense to a 10-point energy spectrum. These values of $\gamma$ are $2.1932,2.1722$, and 2.1634 for $K_{1}, K_{2}$, and $K_{3}$, respectively. Using the same procedure in reverse, the value of $\gamma$ was determined at each of the radial distances at which the pitch angle distributions had been determined. The change in $\gamma$ with decreasing distance is gradual, and $\gamma$ increases by only 0.8 in going from $L=9.5$ to 2.67 .

The value of $k$ at each radial distance can be determined from the integral spectrum using the threshold energy of each detector and the predicted value of $\gamma$. Inside $L=7.5$, the value of $k$ determined from detector A started to decrease relative to that determined from detector B. Every $k$ determined from detector B $(T>560 \mathrm{keV})$ continues to rise in a steady fashion with decreasing distance. If each $k$ determined from detector B is assumed to give the correct spectrum, then the values of $k$ and $\gamma$ can be used to determine 
an effective lower energy cutoff for detector $\mathrm{A}$. The cutoff energy for detector $\mathrm{A}$ increases with decreasing distance inside $L=8$. These results can be interpreted as some process causing a preferential loss of the lowerenergy electrons. The cutoff energy does not correspond to a fixed value of $\mu$, but increases more rapidly with decreasing distance. Inside about $L=5.5$, the cutoff energy increases less rapidly and corresponds to a decreasing value of $\mu$. This is not possible for radial diffusion from an external source; the $\mu$ corresponding to the cutoff energy must remain constant or increase. From a plot of $k$ values determined from detector B for distances greater than $L=5$ versus $\sin ^{2} \alpha_{0} / B_{0}$, it was found that there is a power law dependence of the form $k=D\left(\sin ^{2} \alpha_{0} / B_{0}\right)^{-C}$. Inside this distance, the values of $k$ determined in this fashion increase less rapidly, when compared with this power law dependence. To approximate the differential energy spectrum inside $L=5.5$ that is produced by radial diffusion from an external source, it was assumed that the values of $k$ predicted by the power law dependence outside $L=5.5$ could be extended to smaller distances. This extrapolation inside $L=5.5$ assumes that the effective threshold has become larger than the threshold of detector B (560 keV). An effective cutoff energy corresponding to a constant $\mu_{*}$ was assumed for the data inside $L=5.5$. For the example in Figure 4 , the value of $\mu_{*}$ must be greater than or equal to $470 \mathrm{MeV} / \mathrm{G}$, which corresponds to the maximum value of $\mu$ calculated from the cutoff threshold for detector A. A value of $\mu_{*}=520 \mathrm{MeV} / \mathrm{G}$ was found to give the best overall representation of the data from both detectors for this example. This value is very similar to that found by Van Allen et al. [1980b]. The integral intensity as calculated for the cutoff energy corresponding to this value of constant $\mu_{*}$, the values of $k$ predicted by the power law extrapolation, and the calculated values of $\gamma$ are shown as a solid line in Figure 4 . The solid line, inside $5.5 R_{s}$, represents the upper limit of the integral intensity of electrons diffusing inward from an external source. The integral intensity determined from the measurements of detectors $\mathrm{A}$ and B rises markedly above the solid curve for $L<5$.

The energy spectrum describing all of the electrons inside $5.5 R_{s}$ cannot be determined from these data, since only the spectrum of the inward diffusing electrons can be estimated. The principal conclusion that can be drawn from Figure 4 is that there is an excess of energetic electrons that cannot be explained by radial diffusion from an external source. These excess electrons must be assumed to be from another source. Similar results and conclusions were reached by Krimigis and Armstrong [1982] using the Voyager 2 data. Specifically, they found that inside $L=5$, "there exist a substantial flux of energetic electrons at energies $\geq 1.5 \mathrm{MeV}$ and up to $\sim 20 \mathrm{MeV}^{\prime \prime}$ [Krimigis and Armstrong, 1982, p. 1146] and these particles are locally produced. The other source might be locally produced electrons from cosmic ray interactions with the moons, local ring material, or the $A$ and $B$ rings. This is not unexpected, since there is a large number of energetic protons that have been produced by the decay of neutrons [Blake et al., 1983; Cooper, 1983; Van Allen, 1983]. The source function of electrons must be equal to that of the protons, but their residence times are probably longer if the diffusion processes are the same by virtue of the smaller absorption effect of the satellites [Fillius et al., 1980].

The assumption of a sharp energy cutoff to the energy spectrum is not realistic. Most physical processes such as $d E / d x$ energy loss produce a low-energy tail and a smoothing of the spectrum at low energies. The typical energy loss process produces a low-energy tail proportional to $T^{b}$ where $b$ is of the order of $1.5 \pm 0.2$. The assumed form of the differential energy spectrum of those electrons that have an external source is taken to be

$$
\begin{aligned}
j(T) & =A T^{1.5} & & T<T_{1} \\
& =k T^{-\gamma} & & T>T_{1} .
\end{aligned}
$$

The values of $A$ and $T_{1}$ are determined such that the integral intensity $>40 \mathrm{keV}$ gives the same result as the sharp cutoff energy.



Figure 5. The points are the calculated phase space densities for a pitch angle near $80^{\circ}$. The open circles, squares, triangles, and solid circles are for constant first adiabatic invariants of $125,250,500$, and $1000 \mathrm{MeV} / \mathrm{G}$, respectively. The dashed curves represent fits of the phase space densities, assuming lossy radial diffusion from an external source. The two lower solid curves represent loss-free diffusion with an absorbing boundary at the outer edge of the $A$ ring. The upper curve is for loss-free diffusion inward from $9.5 R_{s}$ and the lower curve is for loss-free diffusion inward from $5.5 R_{s}$. Both of these curves assume that $D_{L L}=D_{0} L^{3}$. 


\section{Phase Space Densities}

Using the energy spectra from the preceding section, the phase space densities of the inward diffusing electrons have been calculated for the three different values of $K$ cited above at four values of $\mu$. The chosen values of $\mu$ were $125,250,500$, and $1000 \mathrm{MeV} / \mathrm{G}$. The discrete points in Figures 5 through 7 show the results for the three values of $K_{i}$. Figure 5 represents the smallest $K$ that gave a complete sampling of the phase space densities inside $10 R_{s}$ to the $A$ ring cutoff. The solid curve near the top of the figure represents loss-free diffusion, assuming $D_{L L}=D_{0} L^{3}$ inward from $9.5 R_{s}$ for $\mu=125 \mathrm{MeV} / \mathrm{G}$, and the higher of the two lower solid curves, that is, the one ending near $L=5.5$ represents the loss-free diffusion for the same particles, assuming their source is at this point. Both curves assume that the phase space density is zero at the outer edge of the $A$ ring. A higher power $L$ dependence of the diffusion coefficient would give higher loss-free diffusion curves.

Figures 5, 6, and 7 all have the same general character. All four phase space density curves show a monotonic decrease with decreasing values of $L$, the relative effect being greater for lesser values of $\mu$. The rapid decrease stops at about $L=5.5$ for the lower three values of $\mu$, because of the assumed form of the spectra of inward diffusing electrons. The $1000-\mathrm{MeV} / \mathrm{G}$ particles seem to be only slightly affected by the lossy region, whereas the densities with lower $\mu$ show strong decreases in this region.

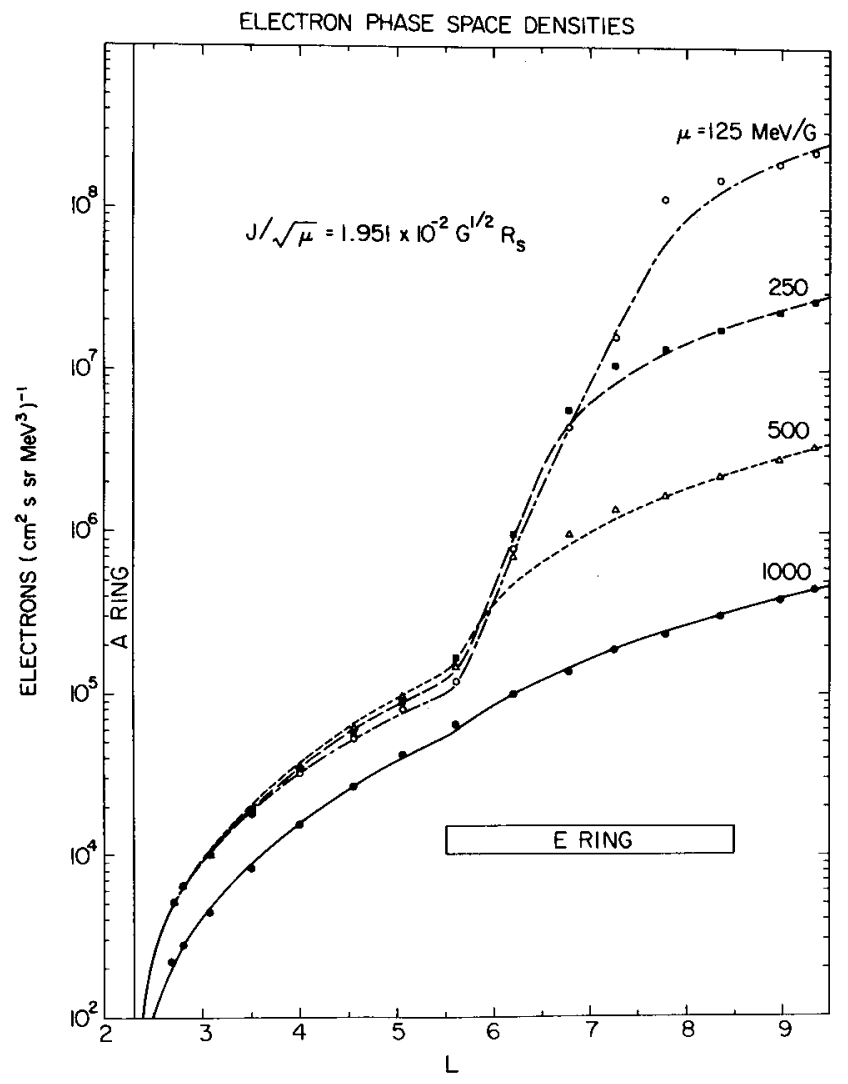

Figure 6. Similar to Figure 5, except for pitch angles near $70^{\circ}$.

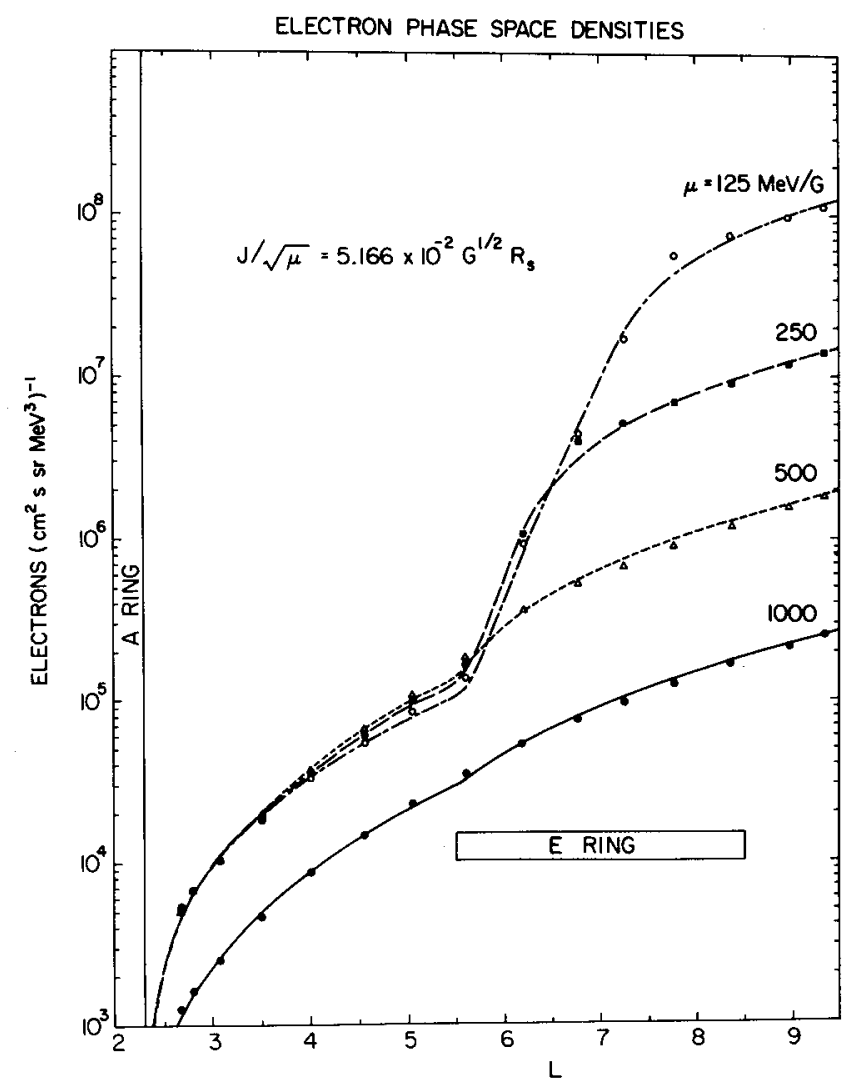

Figure 7. Similar to Figure 5, except for pitch angles near $60^{\circ}$.

It is emphasized that the phase space densities inside $L=5.5$ are for those electrons that have diffused inward from an external source. The observed integral intensities inside $L=5.5$ correspond to substantially greater phase space densities than those inferred in Figures 5-7 for lossy diffusion from an external source. The phase space densities of the excess electrons cannot be easily modeled from the observational data, because the source spectrum is modified by the diffusion process.

Armstrong et al. [1983] constructed phase space densities for electrons with $K^{r}=0.27 G^{1 / 2} R_{s}$ from the Voyager data. These phase space densities showed similar decreases between 5 and $8 R_{s}$ for $\mu=80 \mathrm{MeV} / \mathrm{G}$ and an increase inside $L=4$. Electrons with higher values of $\mu$ inside $L=5$ tended to decrease less rapidly than outside or showed a slight increase. Absolute comparisons with our results are not possible, since they used arbitrary units for the phase space densities and Voyager 2 was at much higher latitudes than Pioneer.

If the low-energy tail approximation was not used for the differential energy spectra, then the phase space densities would consist of only the outer segments. The phase space density for $\mu=125 \mathrm{MeV} / \mathrm{G}$ would become zero between $L=7.5$ and 8 ; and between $L=7.5$ and 5.5 , the phase space densities for both $\mu=250$ and $500 \mathrm{MeV} / \mathrm{G}$ would also drop to zero. Only the highest $\mu=1000 \mathrm{MeV} / \mathrm{G}$ phase space densities would continue to smaller $L$ values.

The phase space densities decrease too strongly in the outer region to be described by loss-free diffusion 
(see Figure 5). From the phase space density plots, it is seen that there is a region of high loss between $L=5.5$ and approximately 8 . But the whole of the inner magnetosphere of Saturn seems to be lossy. If these processes can be characterized by a simple loss term of the form $-f / \tau$, then the reduced radial diffusion equation is

$$
\frac{\partial f}{\partial t}=L^{n} \frac{\partial}{\partial L}\left(\frac{D_{L L}}{L^{n}} \frac{\partial f}{\partial L}\right)-\frac{f}{\tau}+S .
$$

The equation normally used in the literature has $n=2$ and applies where $J=0$. These data were not at the magnetic equator where $J=0$, which is also the case for all of the outer planetary encounters; thus another value should be used. The most appropriate form according to Schulz and Lanzerotti [1974] is $n=5 / 2$. The diffusion coefficient is assumed to be of the form $D_{L L}=D_{0} L^{m}$ and $\partial f / \partial t=0$ for the steady state. The value of $m$ can be determined by several empirical techniques described by Schulz and Lanzerotti. Several models of radial diffusion processes have been developed and these predict values of $m=3,6$, and 10 [Brice and McDonough, 1973; Fälthammar, 1968; Nakada and Mead, 1965]. One of these empirical techniques has been applied to these data, and the corresponding values of $m$ that give the better representation of the phase space densities are in the range of 2 to 4 for $n=5 / 2$.

The adopted technique involves rewriting equation (7), using the above assumptions, in the following form:

$$
D_{0} L^{m} \tau=\frac{f}{\frac{\partial^{2} f}{\partial L^{2}}+\frac{m-5 / 2}{L} \frac{\partial f}{\partial L}} .
$$

Then the first and second derivatives of $f$ are numerically calculated as a function of radial distance. This was done by first fitting In $f$ to a parabola in $L$ using three points and then evaluating the derivatives at the midpoint. The right-hand side of equation (8) can then be evaluated for $m=3,6$, and 10 . This calculated quantity is then plotted on log-log paper versus $L$. If $\tau$ is independent of $L$, then the slope of this curve should be $m$. This process was carried out for all of the phase space densities, and the slopes were all between 2 and 4 , for those data outside $L=7.5$ and inside $L=5.5$, independent of the value of $m$ used in the right-hand side of equation (8). For $m=3$, the slopes were very close to 3 . The data in between showed no such systematic trends, and hence the lifetimes are not constant within this region nor do they have a simple radial dependence. This result does not prove that $m=3$, because it may have a radial dependence of $3-m$, but for the purposes of this paper, $m$ is assumed to be 3 .

\section{Radial Diffusion}

More information from the phase space densities can be extracted by using the following form of the steady state radial diffusion equation:

$$
0=L^{5 / 2} \frac{\partial}{\partial L}\left(\frac{D_{L L}}{L^{5 / 2}} \frac{\partial f}{\partial L}\right)-\frac{f}{\tau}+S .
$$

The source of the particles is taken to be at $L=9.5$, and the outer edge of the $A$ ring is taken as a perfect absorber. The magnetosphere is divided into three parts. The region within which the phase space densities decrease strongly is denoted as region II. The interior region and the exterior region are denoted as I and III, respectively. The diffusion coefficient is assumed to be $D_{L L}=D_{0} L^{3}$ throughout the magnetosphere. And for simplicity, the characteristic lifetimes $\tau_{i}$ are assumed to be different in each region but constant within a given region. This is not true for region II, but the assumption of constancy simplifies the problem. In region I the solution to the diffusion equation is

$$
f_{\mathrm{I}}=\sqrt{L} A \sinh a\left(\frac{1}{\sqrt{L_{0}}}-\frac{1}{\sqrt{L}}\right),
$$

where $L_{0}$ is the outer edge of the $A$ ring and $a=$ $2 /\left(D_{0} \tau_{1}\right)^{1 / 2}$. The solutions in the other two regions are easily found. They contain constants $b, c, L_{1}$, and $L_{2}$, where $b=2 /\left(D_{0} \tau_{2}\right)^{1 / 2}, c=2 /\left(D_{0} \tau_{3}\right)^{1 / 2}$, and $L_{1}$ and $L_{2}$ are the inner radial distances of regions II and III, respectively. These solutions are continuous at each of the regional boundaries, as are their first derivatives. The three expressions for $f$ are not linear and hence are not fittable by any normal least squares techniques. Instead, a different approach was used, namely, $L_{0}$ was set equal to 2.3 and the coefficients $A$ and $a$ were found by varying $a$ and $A$ until the deviations of $\ln (f)$ were minimized using the data inside $L=5.5$. The data from region II were used to determine $L_{1}$ and $b$ in a similar manner. The values of $L_{1}$ were always close to 5.5 , and this value was adopted. The best $b$ was determined for each curve. Similar attempts in region III were not fruitful. $L_{2}$ is not a constant but varies with $\mu$ and $J$ in what seems to be a systematic variation. The best determinations of $L_{2}$ and $c$ were made using the data from region III and the previously determined values of $A, a, b, L_{0}$, and $L_{1}$ for each data set. These determinations are somewhat rougher than the others

Table 1. Coefficients

\begin{tabular}{rrrrrrr}
\hline \multicolumn{2}{c}{$\mu(\mathrm{MeV} / \mathrm{G})$} & $A$ & $a$ & \multicolumn{1}{c}{$b$} & $c$ & $L_{2}$ \\
\hline \multirow{6}{*}{$K_{1}$} & 125 & $3.914 \times 10^{3}$ & 13.1 & 98.7 & 22.0 & 7.90 \\
& 250 & $3.530 \times 10^{3}$ & 14.4 & 100.7 & 10.0 & 6.70 \\
& 500 & $3.086 \times 10^{3}$ & 15.6 & 110.0 & 13.6 & 5.80 \\
& 1000 & $1.972 \times 10^{3}$ & 15.0 & 85.0 & 14.0 & 5.55 \\
$K_{2}$ & 125 & $4.283 \times 10^{3}$ & 12.8 & 101.0 & 11.9 & 7.65 \\
& 250 & $3.860 \times 10^{3}$ & 14.1 & 102.5 & 15.0 & 6.50 \\
& 500 & $3.375 \times 10^{3}$ & 15.2 & 100.0 & 18.7 & 5.70 \\
& 1000 & $1.586 \times 10^{3}$ & 14.4 & 90.0 & 16.0 & 5.54 \\
$K_{3}$ & 125 & $4.351 \times 10^{3}$ & 13.0 & 102.6 & 13.6 & 7.20 \\
& 250 & $3.900 \times 10^{3}$ & 14.2 & 110.0 & 15.4 & 6.15 \\
& 500 & $3.402 \times 10^{3}$ & 15.2 & 100.0 & 16.0 & 6.15 \\
& 1000 & $9.277 \times 10^{2}$ & 14.1 & 100.0 & 17.0 & 5.53 \\
\hline
\end{tabular}

Here $\mu$, in $\mathrm{MeV} / \mathrm{G} ; A$, constant coefficient; $a=$ $2 /\left(D_{0} \tau_{1}\right)^{1 / 2} ; b=2 /\left(D_{0} \tau_{2}\right)^{1 / 2} ; c=2 /\left(D_{0} \tau_{3}\right)^{1 / 2} ; L_{2}$, inner radial distance of region III. 
in regions $I$ and II. The constraints on the coefficients by the interior fits allowed only the asymptotic fit to $f$ in the outer region. These results are shown as the smooth curves in Figures 5 through 7 . The departure of the curves from the data points in the outer region can be seen and is possibly explained by the form of the differential energy spectra used to construct the phase space densities. The various parameters for each "fit" are listed in Table 1.

\section{The $E$ Ring}

\section{Introduction}

The strong decrease of the phase space densities between 8 and $5.5 R_{s}$ is too smooth to be due to satellite interactions. Satellite sweeping would produce more discontinuous changes at the satellite orbits with smooth transitions in between and this is not seen even in the highest time resolution data. Earth-based observations reveal the presence of a faint ring in this region [Feibelman, 1967; Baum et al., 1981]. This ring probably extends inward to near the orbit of Mimas. If the ring particles are responsible for the losses, then it may be possible to say something about the ring particle sizes and their distribution from the dependences of the loss on $\mu$ and $J$, as first suggested by Thomsen and Van Allen [1979].

The time that an electron spends within this region can be estimated from the trans- $L$ diffusional speed [Schultz and Lanzerotti, 1974]:

$$
\dot{L}=-D_{L L}\left(\frac{\partial \ln f}{\partial L}\right)_{\mu, J} .
$$

Rewriting the equation and integrating, the time is found to be

$$
T=\int_{0}^{T} d t=-\int_{L_{b}}^{L_{a}} \frac{f d L}{D_{0} L^{3} \frac{d f}{d L}},
$$

where $f$ can be written as $L^{1 / 2} g$; then

$$
\frac{1}{f} \frac{d f}{d L}=\frac{1}{2 L}+\frac{1}{g} \frac{d g}{d L}
$$

The $1 / 2 L$ term is much smaller than the second term and is neglected. The $L$ dependence of $g$ is an exponential in $-b / L^{1 / 2}$ or $-c / L^{1 / 2}$, depending upon the region of interest. A change of variables $x=b / L^{1 / 2}$ gives

$$
T=\left.\frac{4}{D_{0} b^{2}} \ln \left(\frac{d g}{d x}\right)\right|_{x_{a}} ^{x_{b}}=\left.\tau_{2} \ln \left(\frac{d g}{d x}\right)\right|_{x_{a}} ^{x_{b}} .
$$

The number of collisions that occur during the time period can be calculated and compared to the decrease in the phase space densities. The phase space density at the inner edge of the sharp decrease is compared to that which would occur for loss-free diffusion in order to determine the decrease. The loss-free phase space density was taken to be that which would result from the density at the outer edge diffusing inward and being lost at the outer edge of the $A$ ring. The loss-free phase space density is

$$
f_{L F}=f\left(L_{*}\right) \frac{\sqrt{L}-\sqrt{L_{0}}}{\sqrt{L_{*}}-\sqrt{L_{0}}},
$$

where $L_{*}$ is the outer edge of the $E$ ring. The intensity decrease is

$$
\frac{f\left(L_{1}\right)}{f_{L F}\left(L_{1}\right)}=\frac{f\left(L_{1}\right)}{f\left(L_{*}\right)} \frac{\sqrt{L_{*}}-\sqrt{L_{1}}}{\sqrt{L_{1}}-\sqrt{L_{0}}} .
$$

\section{Thin Ring}

The above ratio must now be related to the diffusion time. Assuming the ring region is thin, the average bounce period $\left\langle\tau_{B}\right\rangle$ can be calculated for each value of $\mu$ and $J$. The number of passes an energetic electron makes through this region is $2 T /\left\langle\tau_{B}\right\rangle$. The amount of material per unit Strärea in the ring is $4 \pi n_{0}\left\langle r_{0}^{3}\right\rangle \rho d / 3$, where $\rho$ is the density, $n_{0}$ is the number of ring particles per unit volume, and $d$ is the thickness of the ring. Here $r_{0}$ is the radius of the ring particles and is a characteristic size of the particle size distribution. Since the data are at several different pitch angles, the path must be corrected by a factor of $\tan \alpha$. The product of all of these terms is $8 \pi n_{0}\left\langle r_{0}^{3}\right\rangle T \rho d \tan \alpha /\left(3\left\langle\tau_{B}\right\rangle\right)$, which has the dimension of mass per unit area. This quantity can be normalized by dividing by the range of the average electron in $\mathrm{g} / \mathrm{cm}^{2}$ for each case. The unknown factors are in the expression $n_{0}\left\langle r_{0}^{3}\right\rangle d / D_{0}$ which is assumed to be constant; hence it is possible to calculate the other quantities and compare them with the intensity decreases.

The outer edge of the $E$ ring is not well known, but the phase space densities indicate that it extends to at least $8 R_{s}$, and the Earth-based observations suggest that it extends as far out as $9 R_{s}$. All quantities were calculated assuming that the outer boundary, $L_{*}$, was at $8 R_{s}, 8.5 R_{s}$, and $9 R_{s}$, respectively. The intensity decreases were plotted versus this calculated quantity, and it was found that the $\tan \alpha$ factor disorganized the data. It was thought that the diffusion coefficient might contain a $\tan \alpha$ factor. A search of the literature showed that this was not the case, but there is a slight dependence on energy for electrons.

\section{Thick Ring}

The other possibility is that the $E$ ring is not thin and that the electrons having pitch angles $\alpha_{0}>60^{\circ}$ spend all their time diffusing within the ring. The areal mass density, normalized by the range of the electrons in this case, is $4 \pi n_{0}\left\langle r_{0}^{3}\right\rangle T \rho d \beta c / 3 R$, where $R$ is the range of the electrons. The average value $\langle\beta / R\rangle$ was calculated using the range energy approximation of Katz and Penfold [1952] and used above. The correlation of this quantity with the fractional decreases in phase space densities using equation (14) was very good, being best for $L=8.5$. For the purposes of the rest of the paper, the outer boundary of the $E$ ring is taken to be 8.5 . No 
effect is actually seen in the observational data outside $L \geq 7.5 R_{s}$.

These results can be interpreted in terms of the transmission of electrons through a given amount of material. The empirical transmission equation of Tabata and Ito [1974] can be used to determine the unknown constants. Their expression for the electron transmission coefficient is

$$
\eta_{T}=\frac{1+\exp ^{-S_{0}}}{1+\exp \left[\left(S_{0}+2\right) \frac{x}{R}-S_{0}\right]},
$$

where $x$ is the thickness, $R$ is range, and $S_{0}$ is a function of the target material and is weakly dependent upon the energy of the electron. For water ice, $S_{0}=3.89$ within $1 \%$ over the energy range of the electrons under consideration. Taking the decrease in phase space densities to be equal to $\eta_{T}$ and using the above value of $S_{0}$, $x / R$ can be solved for in terms of the decreases. Then $x / R$ can be set equal to $W * T\langle\beta / R\rangle$, and the constant $W$ is evaluated to be $6.881 \pm 0.263$ for these data. In terms of the above quantities, $W=4 \pi n_{0}\left\langle r_{0}^{3}\right\rangle \rho c / 3 D_{0}$. Assuming that the $E$ ring is composed of water ice, where $\rho=0.917 \mathrm{~g} / \mathrm{cm}^{3}$ and $c=3 \times 10^{10} \mathrm{~cm} / \mathrm{s}$, then $n_{0}\left\langle r_{0}^{3}\right\rangle / D_{0}=5.97 \times 10^{-11} \pm 2.28 \times 10^{-12}$. If, on the other hand, the $E$ ring is composed of carbonaceouschrondritic material, where we assume $\rho=2.7 \mathrm{~g} / \mathrm{cm}^{3}$, then $n_{0}\left\langle r_{0}^{3}\right\rangle / D_{0}=2.03 \times 10^{-11} \pm 7.77 \times 10^{-13}$.

\section{Comparison With Other Observations}

Smith [1978] estimated the $E$ ring optical thickness from the Earth-based observations of Feibelman to be between $1.0 \pm 0.5 \times 10^{-7}$ and $1.6 \pm 0.8 \times 10^{-6}$ using assumed albedos of 0.8 for icy particles and 0.05 for carbonaceous-chrondrite particles, respectively. The optical thickness is given by $\sigma=\pi n_{0}\left\langle r_{0}^{2}\right\rangle d$, where these quantities have already been defined.

In situ measurements have been made by the Pioneer 11 and Voyager 1 and 2 spacecraft. The Pioneer 11 micrometeoroid detector passed through this region very near the equator and did not observe a single event. The threshold for the detection was $7.1 \times$ $10^{-9} \mathrm{~g}$, or water ice particles with radii greater than $1.23 \times 10^{-3} \mathrm{~cm}$ or carbonaceous-chondrite particles with radii greater than $8.6 \times 10^{-4} \mathrm{~cm}$. A single event would place the particle density at $7.7 \times 10^{-15} / \mathrm{cm}^{3}$, which can be taken as an upper bound for particles of this size. Voyager observations were reported by Gurnett et al. [1983] and Aubier et al. [1983]. Their main emphasis was the detection of particles at the ring plane crossing near the $G$ ring. For completeness, Gurnett et al. listed the average impact rates observed in the occasional wideband data for both Voyager 1 and 2. Some of these data cover the region of interest. The one pertinent Voyager 1 observation was at $6.9 R_{s}$ and at a latitude of $-15.8^{\circ}$, where $10.0 \mathrm{impacts} / \mathrm{s}$ were observed during $48 \mathrm{~s}$ of data. There were 3 wideband frames of data from Voyager 2 in this region. Two consecutive frames at $7.8 R_{s}$ and $24.5^{\circ}$ latitude during the inbound passage gave 0.5 and $1.0 \mathrm{impacts} / \mathrm{s}$, respectively. An ad- ditional frame of wideband data taken on the outbound passage at a distance of $6.4 R_{s}$ and $-25.8^{\circ}$ latitude gave 2.0 impacts/s for the $48 \mathrm{~s}$ of data. The amount of data is meager but tends to verify the assumption that the $E$ ring is geometrically thick.

It is assumed that the $10 \mathrm{impacts} / \mathrm{s}$ is representative of the $E$ ring at lower latitudes and that the rate drops off beyond latitudes of $16^{\circ}$ or so, as indicated by the Voyager 2 data. The impact rate can be interpreted by $I=n_{*} A V$, where $A$ is the projected area of the spacecraft $\left(1.66 \times 10^{4} \mathrm{~cm}^{2}\right), V$ is the relative speed between the spacecraft and the particles $(15.15 \pm 1.35 \mathrm{~km} / \mathrm{s})$, and $n_{*}$ is the particle density above the threshold of observation. Using these values, the number density of ring particles is found to be $n_{*}=(4.00 \pm 0.36) \times 10^{-10} / \mathrm{cm}^{3}$.

A particle size distribution function of the form $n(r) d r=N r^{-3.5} d r$ for $r_{1} \leq r \leq r_{2}$ has been used to describe a fragmentation spectrum [Zuyagaina et al., 1974] and is a plausible function for ring particles. Assuming this distribution for the $E$ ring, $n_{*}$ can be calculated as follows:

$$
n_{*}=\int_{r_{*}}^{r_{2}} n(r) d r=\frac{2 n}{5}\left(r_{*}^{5 / 2}-r_{2}^{-5 / 2}\right) .
$$

Gurnett et al. [1983] made a determination of $r_{*}$ at very high impact rates (i.e., 500/s), and later Gurnett et al. [1987] made some changes in their estimation and procedure for the determination of $r_{*}$ at Neptune. For this situation at Saturn, the impact rate is very low and the 16-channel analyzer outputs are very near background [Scarf et al., 1983]. The wideband data are only a relative measure, since the output has an automatic gain control which is inversely proportional to the rms output voltage. Since these data were acquired during quiet times, the output should be near its lowest level. The thresholds for the 16-channel analyzer are between 1 and $2 \times 10^{-5} \mathrm{~V}$ [Scarf et al., 1981]. Assuming that the wideband data have a similar threshold, 4 times this value should represent the threshold voltage for detection of a particle (L. Granroth, private communication, 1990). The corresponding radii are 7.3 to $9.2 \times 10^{-5} \mathrm{~cm}$ for water ice particles and 5.1 to $6.4 \times 10^{-5} \mathrm{~cm}$ for carbonaceous-chrondrite particles. An estimate of $n_{0}\left\langle r_{0}^{3}\right\rangle$ can be made using these values, the measured number density, the optical thickness, and the assumed particle size distribution function. It is easy to show from the distribution function that $n_{0}\left\langle r_{0}^{3}\right\rangle=n_{0}\left\langle r_{0}^{2}\right\rangle\left(r_{1} r_{2}\right)^{1 / 2}$, and assuming the thickness of the ring to be of the order of $3 R_{s}$, we take $d=2 \times 10^{10} \mathrm{~cm}$. Then $n_{0}\left\langle r_{0}^{2}\right\rangle$ can be approximated from the optical thickness to be equal to $1.59 \pm 0.79 \times 10^{-18}$ for icy particles and $2.55 \pm 1.27 \times 10^{-17}$ for carbonaceouschrondritic particles. For the later type of particles, taking $r_{2}=8.6 \times 10^{-4}$ and the values of $r_{*}$, the value of $N$ can be determined from equation (16). The range of values of $r_{1}$ can then be determined from $n_{0}\left\langle r_{0}^{2}\right\rangle$. The distribution of sizes appears to be wide, and the values of $r_{1}$ are of the order of $10^{-6} \mathrm{~cm}$. This gives a range of values for $\left(r_{1} r_{2}\right)^{1 / 2}=2.5 \times 10^{-5}$ to $1.37 \times 10^{-4} \mathrm{~cm}$. The values of $r_{1}$ are. probably too low for these particles to 
have long-term stability because of electromagnetic and Poynting-Robertson effects. Another approach is to assume that the distribution is very narrow. In this case the range of values for $\left(r_{1} r_{2}\right)^{1 / 2}$ is 1.6 to $3.2 \times 10^{-4} \mathrm{~cm}$. For icy particles, the size distribution has to be nearly delta function and the value of radii of these particles is between 4.3 and $8.1 \times 10^{-5} \mathrm{~cm}$. The lower limit is below the threshold of detection estimated for the Gurnett et al. observations, but it can be taken to give a lower limit to the value of $D_{0}$.

The value for the diffusion coefficient $D_{0}$ can now be estimated from the absorption to be in the range of $1.5 \times 10^{-11}$ to $6.3 \times 10^{-10} R_{s}^{2} / \mathrm{s}$, if the $E$ ring material is carbonaceous-chrondrite with an albedo of 0.05 and in the range of $5.6 \times 10^{-13}$ to $3.4 \times 10^{-12} R_{s}^{2} / \mathrm{s}$, if the material is water ice with an albedo of 0.8 .

\section{Cosmic Ray Albedo Neutron Decay}

As an early explanation for the high-energy protons in the inner magnetosphere of Saturn, a number of authors suggested that their source was from the decay of neutrons produced by cosmic rays hitting the rings of Saturn [Fillius et al., 1980]. Several order of magnitude calculations showed that to be a possibility, if the value of the diffusion coefficient were lower than then assumed. Detailed calculations on some aspects of this problem were given by Blake et al. [1983]. Their paper contains much valuable information about possible energy spectra of the neutrons and their yield per incident cosmic ray.

For every decaying neutron which produces a highenergy proton, there is an associated energetic electron which can also be trapped in the magnetic field. Such electrons may be responsible for the excess of electrons in the inner magnetosphere, as suggested by the previous diffusional calculations. To check out this possibility, the source strength of cosmic ray albedo neutron decay (CRAND) electrons was calculated using the procedure outlined by Nakada [1963]. This procedure assumes that all electrons with kinetic energy $T^{\prime}$ are emitted isotopically in the rest frame. Upon being transformed to the laboratory frame of reference, the energy distribution of electrons with energy $T$ in the rest frame will be constant between the minimum and maximum transformed energies. Small elements of the spectrum from the neutron decay are relativistically transformed to the laboratory frame to produce the electron source spectrum. The calculation was made for the total area of the $A$ and $B$ rings. The neutron spectrum and yield calculated by Blake et al. [1983] for $20 \mathrm{GeV}$ protons on $200-\mathrm{cm}$ ice spheres were used as the source spectrum for the electrons. The time derivative of the differential electron spectrum is equal to the differential neutron spectrum times the injection factor divided by the product of the Lorentz factor and the lifetime of the neutron. Time dilation gives approximately a constant decay rate throughout the magnetosphere. The cosmic ray production of the neutrons was calculated for both the $A$ and $B$ rings above the Störmer cutoff energy. The differential energy spectrum of the cosmic rays is taken

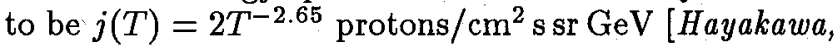
1969].

The electron source strength was calculated from 0.01 to $10 \mathrm{MeV}$ in $10-\mathrm{keV}$ increments, using the neutron spectrum between $10 \mathrm{MeV}$ and $94 \mathrm{GeV}$. Neutrons below $10 \mathrm{MeV}$ were excluded, since very few would get into the trapping region before they decayed. Neutrons with energies greater than $94 \mathrm{GeV}$ will not produce any electrons with energies less than $10 \mathrm{MeV}$. The spectra were normalized such that the number density of electrons was equal to that of the neutrons greater than $10 \mathrm{MeV}$. The injection coefficient for the electrons was calculated for every $0.05 R_{s}$, from the outer edge of the $A$ ring to $10 R_{s}$ and for every $5^{\circ}$ of pitch angle. The electrons were assumed to be injected isotopically along a flux tube, and the occultation of the rings by the planet was taken into account in the calculations. The calculation of the injection coefficient involved the integration of the radial dependence of the Störmer cutoff energy divided by the square of the distance from the source point in the rings to the injection site over the area of both the $A$ and $B$ rings that are visible at the injection site. The calculation was normalized by dividing by the area. The results were calculated at a number of latitudes along the field line corresponding to every $5^{\circ}$ of equatorial pitch angle. These values were adjusted for the change in differential volume at each point along the flux tube, and the total injected intensity at a given pitch angle was found by integrating between the mirror points. From these two separate calculations, the source strength as a function of $L, \mu$, and $J$ was obtained by interpolation.

With an internal source, which is a function of $L$, the steady state diffusion equation (9) has to be treated as a nonhomogeneous second-order differential equation and has a specific solution which includes the internal source term and a homogeneous solution that does not include the internal source term. The specific solution to the diffusion equation is

$$
\begin{aligned}
f_{s}= & \frac{\sqrt{L}}{D_{0} a}\left[\exp ^{a / \sqrt{L}} \int_{L_{0}}^{L} \frac{S(x) d x \exp ^{-a / \sqrt{x}}}{x^{2}}\right. \\
& \left.-\exp ^{-a / \sqrt{L}} \int_{L_{0}}^{L} \frac{S(x) d x \exp ^{a / \sqrt{x}}}{x^{2}}\right]
\end{aligned}
$$

where $a=2 /\left(D_{0} \tau_{1}\right)^{1 / 2}$. The general solution for the phase space density is the sum of the specific and homogeneous solutions. The homogeneous solution is

$$
f_{h}=A \sqrt{L} \exp ^{a / \sqrt{L}}+B \sqrt{L} \exp ^{-a / \sqrt{L}} .
$$

The boundary conditions for the general solution are that $f=0$ at the outer edge of the $A$ ring and that $f$ is equal to the observed phase space density at some distant point.

Dividing the Saturnian magnetosphere into three regions as before, with three different loss rates, places the same type of conditions on $f_{s}$ as were required be- 
fore. The functions and their first derivatives must be continuous across each boundary between the three regions. Solving these equations, subject to the above conditions at each boundary and integration by parts, allows $f_{s}$ to be computed for all regions.

In order to simplify the calculations, the average values of $a, b$, and $c$ were used. The outer edge of the $A$ ring was assumed to be at $L=2.3$, and the inner edge of the absorbing region was assumed to be at $L=5.5$. The outer edge of influence of the $E$ ring was again allowed to vary with energy. The source strength divided by $L^{2}$ was computed at every $0.05 L$ for constant $\mu$ and $J$ corresponding to the previous analysis. These functions were integrated numerically using Simpson's rule after multiplying by the exponential factors. The specific solution was obtained at every tenth of an $L$. The function was not divided by $D_{0}$, since it was unknown and was only a multiplicative factor.

The total phase space density is the sum of this function and the previously defined $f$ as given in equation (10). The arbitrary factor $A$ in equation (10) can now be determined by the outer boundary condition. The condition chosen was that the calculated phase space density be equal to that value of the phase space density determined from the fits to the data at $L=9$.

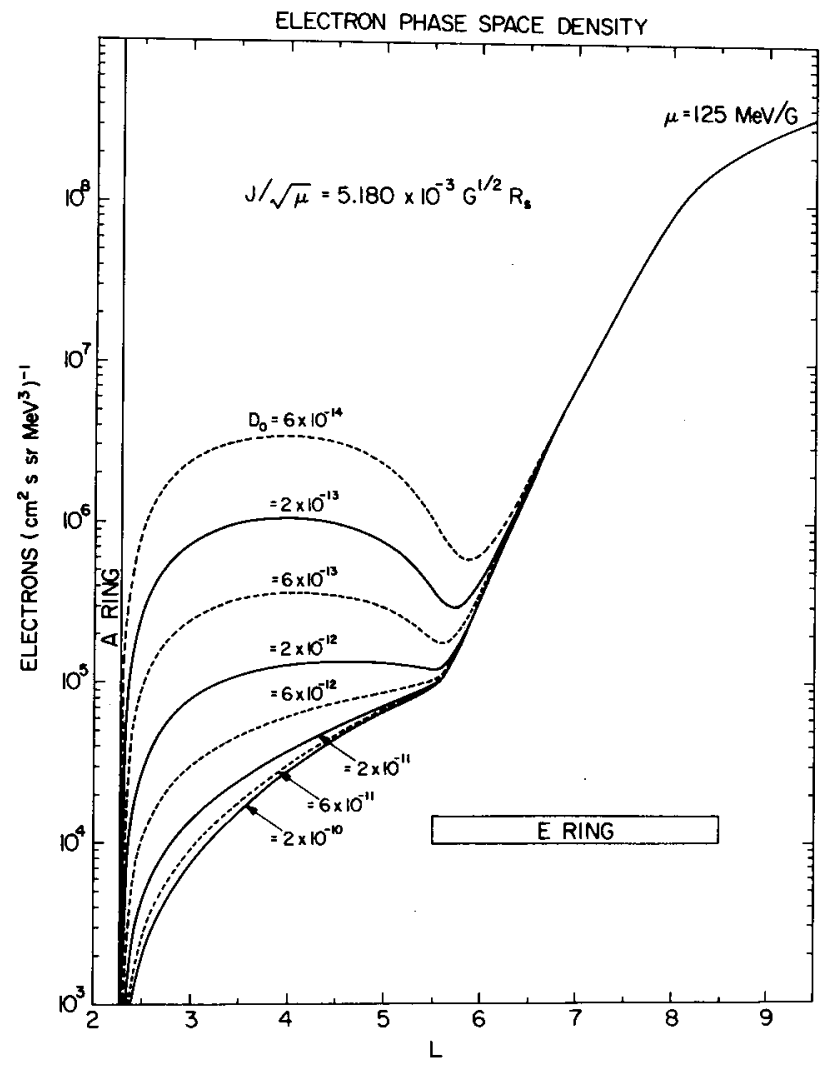

Figure 8. Calculated phase space densities for pitch angles near $80^{\circ}$ and for $\mu=125 \mathrm{MeV} / \mathrm{G}$. These curves combine radial diffusion from an external source with an internal cosmic ray albedo neutron decay (CRAND) source. The value of the diffusion coefficient is the only variable. The curves are normalized to the phase space density at $9 R_{s}$.

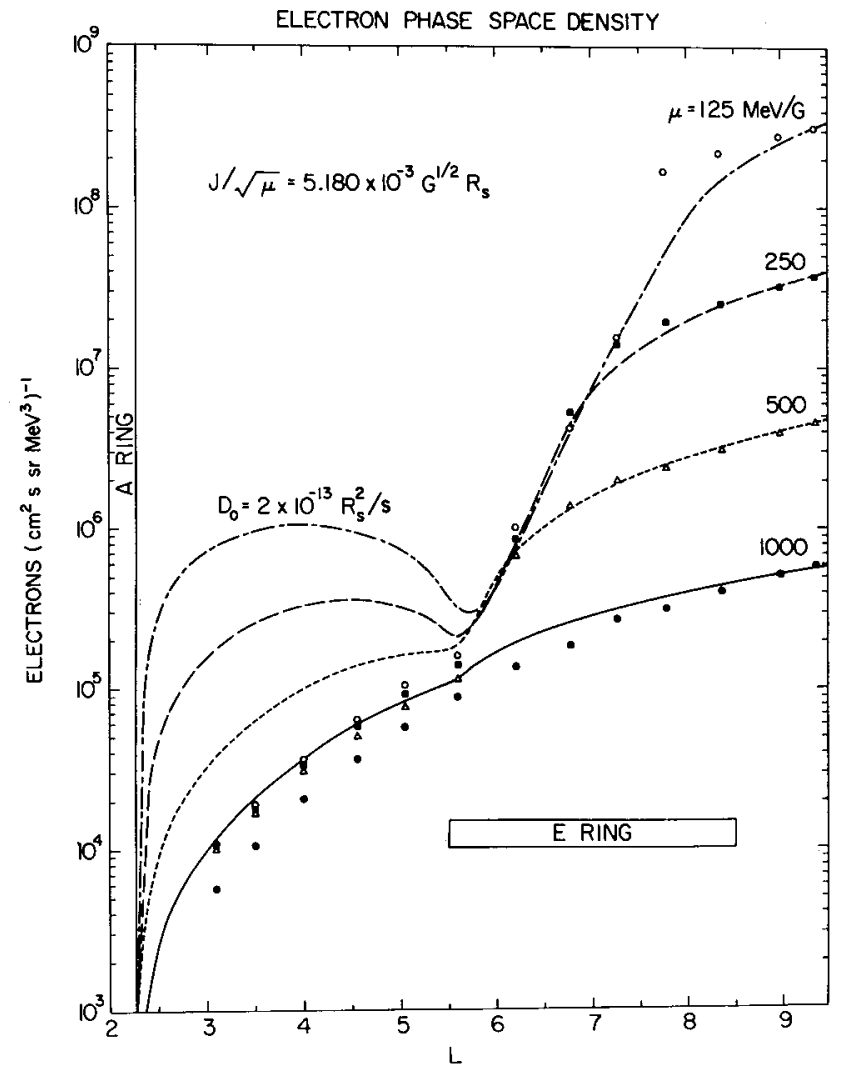

Figure 9. Similar to Figure 8, but includes the previously calculated phase space densities and all four values of $\mu$. This is for $D_{0}=2 \times 10^{-13} R_{s}^{2} / \mathrm{s}$.

Thus for a given value of $D_{0}$, the value of $A$ can be determined for each set of $\mu$ and $J$.

Starting with $D_{0}=6 \times 10^{-11} R_{s}^{2} / \mathrm{s}$, which was near the upper limit determined from the absorption data, the calculated phase space densities were found to be inadequate to explain the intensities observed by detectors $\mathrm{A}$ and $\mathrm{B}$ in the inner region. The value of $D_{0}$ was lowered progressively until there were approximately enough particles in the inner region to explain the observed integral intensities of electrons. Figure 8 shows the results of the progressive lowering of the value of $D_{0}$ for the case of $K=5.180 \times 10^{-3} G^{1 / 2} R_{s}$ and $\mu=125 \mathrm{MeV} / \mathrm{G}$. The differential energy spectra were calculated at $L=3,3.5,4$, and 4.5 for the various values of $D_{0}$ from the phase space densities. These were then integrated numerically to obtain the integral flux greater than $0.56 \mathrm{MeV}$. The values compare well with the observed intensities of detector $\mathrm{B}$ at $L=3$ and 3.5 for $D_{0}=2 \times 10^{-13}$ and at $L=4$ and 4.5 for $D_{0}=4$ and $6 \times 10^{-13} R_{s}^{2} / \mathrm{s}$. The discrepancy in not finding a constant value of $D_{0}$ at $L=4$ and 4.5 probably is indicative of the $E$ ring extending inward to at least $L=4$. Figure 9 shows the phase space densities for the same value of $K$ and for $\mu=125,250,500$, and $1000 \mathrm{MeV} / \mathrm{G}$ using $D_{0}=2 \times 10^{-13} R_{s}^{2} / \mathrm{s}$. The phase space densities that were originally calculated from the data, assuming that they were only from an external source, are also plotted for reference. 

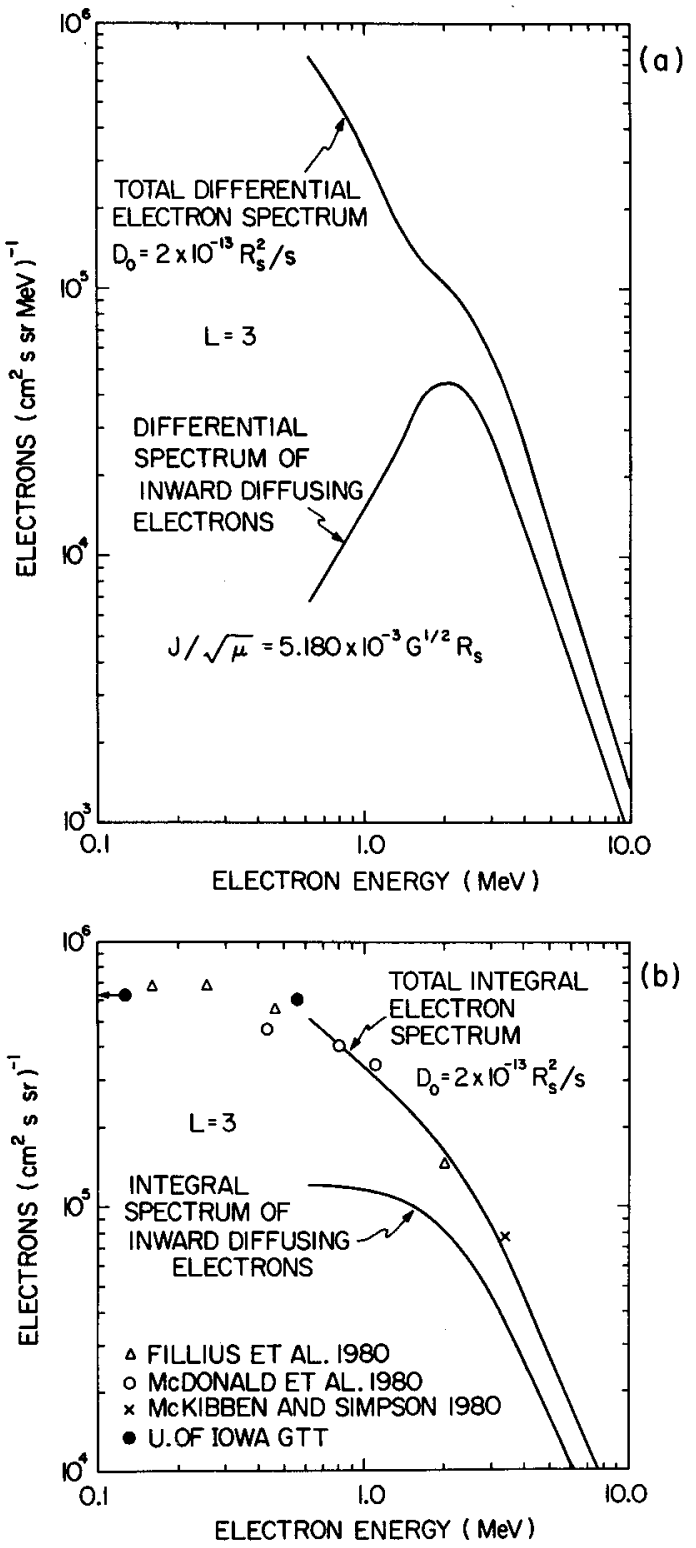

Figure 10. (a) The total differential electron spectrum at $L=3$, calculated from the phase space densities shown in Figure 9. The lower differential energy spectrum is calculated from the phase space densities, assuming that there is no CRAND source of electrons. (b) The integral electron spectrum found by numerical integration of the differential energy spectra shown in Figure 10a. Observations of integral electron intensities by all four energetic particle experiments on Pioneer 11 are shown for comparison.

Figure 10a shows the resulting electron differential energy spectra calculated from the phase space densities in Figure 9 at $L=3$. The higher curve shows the sum of the CRAND and inward diffusing electron spectra. The lower spectrum represents only those electrons that have diffused inward from the outer edge of the magnetosphere. Figure 10b shows the integrals of these two spectra. Observed integral electron intensities from the various Pioneer 11 experiments are shown as discreet points. The observed data from our experi- ment (University of Iowa GTT) and from Fillius et al. [1980] are near $90^{\circ}$ pitch angle, while the other data are spin-averaged observations. The lower energy of all spectra corresponds to $\mu=125 \mathrm{MeV} / \mathrm{G}$. The phase space densities shown in Figure 9 are too high in the region between $L=4$ and 5.5 to account for the observed integral intensities. To have a constant value of $D_{0}$ and to also account for the observed integral intensities, the effects of the $E$ ring must be extended inside $L=5.5$. This refinement would necessitate recalculating everything, starting with the inward diffusing electrons, and is not essential to the principal result. The basic point is that in order to explain the observed integral intensities of electrons in terms of the calculated CRAND source in the rings of Saturn, the value of $D_{0}$ is of the order of $2 \times 10^{-13} R_{s}^{2} / \mathrm{s}$. If the calculated CRAND source strength were higher, then the corresponding value of $D_{0}$ would be higher by the same amount.

Van Allen [1983] found that the energetic protons in the inner magnetosphere of Saturn could be explained in terms of CRAND-generated protons from the rings. By simple arguments, he found that $S / D=6.9 \times$ $10^{-24} \mathrm{~cm}^{-5}$ at $2.67 R_{s}$. He then used the results of Blake et al. [1983] to estimate that $S=3.3 \times 10^{-15} / \mathrm{cm}^{3} \mathrm{~s}$. A simple calculation gives $D=1.33 \times 10^{-11} R_{s}^{2} / \mathrm{s}$ at $2.67 R_{s}$ and, assuming $D_{L L}=D_{0} L^{3}$, implies that $D_{0}=7 \times 10^{-13} R_{s}^{2} / \mathrm{s}$.

The distribution of ring particle sizes in the $A$ and $B$ rings has not been determined to any great accuracy, but several estimates place the range between a few centimeters and as high as $50 \mathrm{~m}$, with the bulk of the particles near the low end of this range [Marouf et al., 1982]. Thus the use of $200-\mathrm{cm}$ ring particles in the above calculations makes these determinations of the diffusion coefficient a lower limit. The yield of neutrons produced by cosmic rays hitting several small particles can be as much as 10 times greater than the yield from just one collision with a larger particle [Blake et al., 1983].

Van Allen's estimate of $S$ should be raised by a factor of 10 to account for the increased yield of neutrons from smaller ring particles. Blake et al. [1983] assumed that the production of neutrons takes place everywhere in the $A, B$, and $C$ rings. The $C$ ring is probably too thin to make any significant contribution. Thus the estimate of $S$ should also be decreased by $20 \%$ to account for the decreased area. These changes to Van Allen's estimate then give $D_{0}=5.6 \times 10^{-12} R_{s}^{2} / \mathrm{s}$ for the energetic protons. By the same reasoning, the value of $D_{0}$ obtained for the CRAND electrons should be raised by a factor of 10 to account for the increase of the source strength from smaller ring particles. This gives a value of $D_{0}=2 \times 10^{-12} R_{s}^{2} / \mathrm{s}$ for the CRAND electrons, which is in the range of the estimates from the absorption by icy particles in the outer $E$ ring.

The lower limits placed upon the diffusion coefficient for low-energy ions by Paonessa and Cheng [1986] and for electrons by Chenette and Stone [1983] would eliminate both the CRAND process as the source of the majority of the electrons in the inner region of Saturn's magnetosphere and also the absorption analysis and are 
therefore incompatible with the present analysis. The cited authors also dismiss the determinations of the radial diffusion coefficient by Van Allen et al. [1980a, b], Krimigis et al. [1981], Van Allen [1982, 1983], and Cooper [1983]. These differences are due to the analysis of microsignatures and ascribing the fill-in times to radial diffusion. This is probably wrong, since the gross radial diffusion theory assumes that the phase space densities are longitudinally averaged and the microsignatures are discrete in longitude. The electron microsignature analysis of Chenette and Stone arrives at a lower limit to the diffusion coefficient using the procedure of Van Allen et al. [1980c], which gave an upper limit to the diffusion coefficient. Their analysis also disagrees with the original assessment by Vogt et al. [1982], that these observations were due to pileup of lower-energy electrons. The fact that their estimate of the flux of $>2.5-\mathrm{MeV}$ electrons is several orders of magnitude lower than the expected flux, even assuming an efficiency of $10 \%$, has lead us to reevaluate the Vogt et al. [1982] assumptions. We find that the observations are consistent with a twofold pileup of approximately $1.6-\mathrm{MeV}$ electrons in detector $\mathrm{B} 2$ and threefold or fourfold pileup in the higher-energy detectors. This accounts for the deeper signature in the coincidence channel. The real signature after correcting for dead time is only a $20 \%$ depression in the counting rate, and according to Vogt et al., this signature is of the order of 30 hours old. Details of this detector system, dead time corrections and coincidences are discussed in the paper by Schardt and McDonald [1983].

The lower limit of the diffusion coefficient for lowenergy ions found by Paonessa and Cheng [1986] is based on observations of ions, presumed to be oxygen, in the energy range of 87 to $434 \mathrm{keV}$. These particles are at a nominal equatorial pitch angle of $35^{\circ}$. Their lower limit to the diffusion coefficient was found by finding the minimal value of $D$ for the flux to be zero after crossing the geometrical sweeping region of each satellite using their calculated sweeping lifetimes. This assumes that the sweeping is the greatest loss mechanism in the inner magnetosphere of Saturn, that pitch angle scattering of equatorial energy degraded particles does not contribute to the observed intensities, and that the observations are of low-energy ions. The latter question is raised because of the observations of Carbary et al. [1983]. They see the same microsignature as observed in the high-energy electrons, but the microsignature occurs in the same ion channels from which the phase space densities were derived. Carbary et al. present an argument that only a maximum of $9 \%$ of the $20 \%$ decrease in the ion channels could be attributed to highenergy electrons. But the coincidence of simultaneous and almost identical decreases in high-energy electrons $\geq 1.5 \mathrm{MeV}$ and low-energy ions due to a 30 -hour-old encounter with Mimas is hard to understand.

Armstrong et al. [1983], using the same ion data as Paonessa and Cheng, concluded that inside $L=5$, the ions were locally produced and had not arrived via radial diffusion from an external source.
In view of these arguments, the low value of $D_{0}=2 \times 10^{-12} R_{s} / \mathrm{s}$ is plausible, and CRAND electrons might explain the excess of high-energy electrons that we and Krimigis et al. [1981] and Armstrong et al. [1983] report.

\section{Conclusions}

The inner magnetosphere $(L<10)$ of Saturn is stable but lossy for energetic electrons. The mechanism for their radial diffusion has the same $L$ dependence $\left(D_{L L}=D_{0} L^{3}\right)$ as that proposed by Brice and $M c$ Donough [1973] for Jupiter. In the region $5.5<L<8.5$, the losses are due to collisions with $E$ ring particles. Satellite absorption has a negligible effect on the energetic electrons.

The $E$ ring has a latitudinal thickness on the order of $3 R_{s}$, and assuming the particle size distribution is very narrow, the mean radii of the particles in this ring are in the range of $4 \times 10^{-5}$ to $3.2 \times 10^{-4} \mathrm{~cm}$. The value of the diffusion coefficient $D_{0}$ can be estimated from the absorption to be in the range of $1.5 \times 10^{-11}$ to $6.3 \times 10^{-10} R_{s}^{2} / \mathrm{s}$, if the $E$ ring material is carbonaceous-chrondrite and in the range of $5.6 \times 10^{-13}$ to $3.4 \times 10^{-12} R_{s}^{2} / \mathrm{s}$, if the ring material is water ice.

The observed intensities of energetic electrons inside the $E$ ring are too great to be explained by radial diffusion from an external source. CRAND electrons resulting from cosmic ray interactions in the $A$ and $B$ rings are a quantitatively plausible explanation for the excess. The diffusion coefficient necessary for the CRAND electrons to explain the excess intensities in the inner magnetosphere of Saturn is of the order of 1 to $3 \times 10^{-12} R_{s} / \mathrm{s}$. This range is consistent with the determination from the absorption analysis for the $E$ ring to be composed of water ice particles.

\section{Appendix}

Using relations (1), (2), and (3),

$$
\begin{aligned}
\frac{R(\alpha)}{\text { detector area }}= & J_{0} A_{00}+J_{2}\left(A_{20} \cos ^{2} \beta+A_{21}\right) \\
& +J_{4}\left(A_{40} \cos ^{4} \beta\right. \\
& \left.+A_{41} \cos ^{2} \beta+A_{42}\right)+\cdots
\end{aligned}
$$

where the coefficients $A_{n m}$ are constants.

$$
A_{00}=2 \pi \int_{0}^{\theta_{0}} \sin \theta d \theta F(\theta)=2 I_{0}
$$

The directional geometric factor of the detector is the area of the detector times $A_{00}$. If

then

$$
I_{2 N}=\pi \int_{0}^{\theta_{0}} \sin \theta F(\theta) d \theta \cos ^{2 N} \theta,
$$

$$
\begin{aligned}
& A_{00}=2 I_{0} \\
& A_{20}=3 I_{2}-I_{0}
\end{aligned}
$$




$$
\begin{aligned}
& A_{21}=I_{0}-I_{2} \\
& A_{40}=\left(35 I_{4}-30 I_{2}+3 I_{0}\right) / 4 \\
& A_{41}=\left(18 I_{4}-15 I_{2}-3 I_{0}\right) / 2 \\
& A_{42}=3\left(I_{0}-2 I_{2}+I_{4}\right) / 4, \text { etc. }
\end{aligned}
$$

In addition, the smear due to the finite sampling time has to be taken into account. To do this, it is necessary to integrate all terms over the roll angle from $\eta-\Delta$ to $\eta+\Delta$, where $2 \Delta$ is the angle at which the detector moved during the sampling period. For the Pioneer 11 detectors, the look directions are orthogonal to the spin axis, thus the pitch angle $\beta$ is given by

$$
\cos \beta=\sin \Gamma \cos (\chi-\eta) \text {. }
$$

The general relationship between these vectors is shown in Figure $1 \mathrm{~b}$ and is

$$
\cos \beta=\cos \lambda \cos \Gamma+\sin \lambda \sin \Gamma \cos (\chi-\eta)
$$

where $\lambda$ is the angle between the detector look direction and the spin axis, $\Gamma$ is the magnetic cone angle, and $\chi$ is the magnetic clock angle. For the case where $\lambda=90^{\circ}$, the results are straightforward.

$$
\begin{gathered}
\int_{\eta-\Delta}^{\eta+\Delta} d \eta=2 \Delta=C_{00} \\
\int_{\eta-\Delta}^{\eta+\Delta} \cos ^{2} \beta d \eta=C_{20} \cos ^{2} \beta+C_{21} \sin ^{2} \Gamma \\
\int_{\eta-\Delta}^{\eta+\Delta} \cos ^{4} \beta d \eta= \\
C_{40} \cos ^{4} \beta+C_{41} \sin ^{2} \Gamma \cos ^{2} \beta \\
+C_{42} \sin ^{4} \Gamma .
\end{gathered}
$$

The coefficients are

$$
\begin{aligned}
& C_{20}=2 \sin \Delta \cos \Delta \\
& C_{21}=\Delta-\sin \Delta \cos \Delta \\
& C_{40}=\left(2 \cos ^{2} \Delta-1\right) 2 \cos \Delta \sin \Delta \\
& C_{41}=4 \sin ^{3} \Delta \cos \Delta \\
& C_{42}=\left(3 \Delta-\left(5-2 \cos ^{2} \Delta\right) \cos \Delta \sin \Delta\right) / 4 .
\end{aligned}
$$

These results can be combined to produce the final relationship:

$$
\begin{aligned}
\frac{R(\alpha)}{\text { detector area }}= & J_{0} A_{00} C_{00}+J_{2} A_{21} C_{00} \\
& +J_{4} A_{42} C_{00}+J_{2} A_{20} C_{20} \cos ^{2} \beta \\
& +J_{2} A_{20} C_{21} \sin ^{2} \Gamma \\
& +J_{4} A_{41} C_{20} \cos ^{2} \beta \\
& +J_{4} A_{41} C_{21} \sin ^{2} \Gamma \\
& +J_{4} A_{40} C_{40} \cos ^{4} \beta \\
& +J_{4} A_{40} C_{41} \sin ^{2} \Gamma \cos ^{2} \beta \\
& +J_{4} A_{40} C_{42} \sin ^{4} \Gamma+\cdots
\end{aligned}
$$

$$
\begin{aligned}
= & \left(J_{0} A_{00} C_{00}+J_{2} A_{21} C_{00}+J_{4} A_{42} C_{00}\right. \\
& +J_{2} A_{20} C_{21} \sin ^{2} \Gamma+J_{4} A_{41} C_{21} \sin ^{2} \Gamma \\
& \left.+J_{4} A_{40} C_{42} \sin ^{4} \Gamma+\cdots\right) \\
& +\cos ^{2} \beta\left[J_{2} A_{20} C_{20}+J_{4} A_{41} C_{20}\right. \\
& \left.+J_{4} A_{40} C_{41} \sin ^{2} \Gamma+\cdots\right] \\
& +\cos ^{4} \beta\left[J_{4} A_{40} C_{40}+\cdots\right]+\cdots .
\end{aligned}
$$

The deconvolution process is as follows: The observed data are fit via least squares, with a polynomial of the form $R(\beta(\alpha))=B_{0}+B_{2} \cos ^{2} \beta+B_{4} \cos ^{4} \beta \ldots$. Next, the highest-order term is solved for to yield $J_{4}=B_{4} /$ (Area* $\left.A_{40} C_{40}\right)$. The next term is

$$
J_{2}=\frac{B_{2}}{\left(\text { Area } * A_{20} C_{20}\right)}-\frac{J_{4}\left(A_{41} C_{20}+A_{40} C_{41} \sin ^{2} \Gamma\right)}{\left(A_{20} C_{20}\right)}
$$

and the last is

$$
\begin{aligned}
J_{0}= & \frac{B_{0}}{\left(\text { Area } * A_{00} C_{00}\right)}+\frac{J_{2}\left(A_{21} C_{00}+A_{20} C_{21} \sin ^{2} \Gamma\right)}{\left(A_{00} C_{00}\right)} \\
& -\frac{J_{4}\left(A_{42} C_{00}+A_{41} C_{21} \sin ^{2} \Gamma+A_{40} C_{42} \sin ^{4} \Gamma\right)}{\left(A_{00} C_{00}\right)}
\end{aligned}
$$

Acknowledgments. The author wishes to thank J. A. Van Allen for his critical reading and commenting on this paper. Also thanks to Alice Shank for $\mathrm{T}_{\mathrm{E} X i n g}$ this paper, John Birkbeck for the graphics, and finally I would like to thank one of the referees for several critical comments that have greatly improved this paper. This work was supported by National Aeronautics and Space Administration/Ames Research Center grant NAG 2-571, by the U.S. Office of Naval Research grant N00014-89-J-1179, and by NASA grant NAGW-1739.

The Editor thanks D. L. Gallagher and another referee for their assistance in evaluating this paper.

\section{References}

Armstrong, T. P., M. T. Paonessa, E. V. Bell II, and S. M. Krimigis, Voyager observations of Saturnian ion and electron phase space densities, J. Geophys. Res., 88, 88938904, 1983.

Aubier, M. G., N. Meyer-Vernet, and B. M. Pedersen, Shot noise from grain and particle impacts in Saturn's ring plane, Geophys. Res. Lett., 10, 5-9, 1983.

Baum, W. A., T. Kreidl, J. A. Westphal, G. E. Danielson, P. K. Seidelmann, D. Pascu, and D. G. Currie, Saturn's $E$ ring 1: CCD observations of March 1980, Icarus, 47 , 84-96, 1981.

Blake, J. B., H. H. Hilton, and S. H. Margolis, On the injection of cosmic ray secondaries into the inner Saturnian magnetosphere, 1, Protons from the CRAND process, J. Geophys. Res., 88, 803-807, 1983.

Brice, N. M., and T. R. McDonough, Jupiter's radiation belts, Icarus, 18, 206-219, 1973.

Carbary, J. F., S. M. Krimigis, and W.-H. Ip, Energetic particle microsignatures of Saturn's satellites, J. Geophys. Res., 88, 8947-8958, 1983.

Chenette, D. L., and E. C. Stone, The Mimas ghost revisited: An analysis of the electron flux and electron microsigna- 
tures observed in the vicinity of Mimas at Saturn, J. Geophys. Res., 88, 8755-8764, 1983.

Cooper, J. F., Nuclear cascades in Saturn's rings: Cosmic ray albedo neutron decay and origins of trapped protons in the inner magnetosphere, J. Geophys. Res., 88, 39453954, 1983.

Davis, L., Jr., and E. J. Smith, New models of Saturn's magnetic field using Pioneer 11 vector helium magnetometer data, J. Geophys. Res., 91, 1373-1380, 1986.

Fälthammar, C.-G., Radial diffusion by violation of the third adiabatic invariant, in Earth's Particles and Fields, edited by B. M. McCormac, pp. 157-169, Reinhold, New York, 1968.

Feibelman, W. A., Concerning the $\mathrm{D}^{\prime}$ ring of Saturn, Nature, 214, 793-794, 1967.

Fillius, W., W. H. Ip, and C. E. Mcllwain, Trapped radiation belts of Saturn: First look, Science, 207, 425-431, 1980.

Gurnett, D. A., E. Grün, D. Gallagher, W. S. Kurth, and F. L. Scarf, Micron-sized particles detected near Saturn by Voyager plasma wave instrument, Icarus, 53, 236-254, 1983.

Gurnett, D. A., W. S. Kurth, F. L. Scarf, J. A. Burns, J. N. Cuzzi, and E. Grün, Micron-sized impacts detected near Uranus by Voyager 2 plasma wave instrument, J. Geophys. Res., 92, 14,959-14,968, 1987.

Hayakawa, S., Cosmic Ray Physics, John Wiley, New York, 1969.

Katz, L., and A. S. Penfold, Range-energy relations for electrons and the determination of beta-ray end-point energies by absorption, Rev. Mod. Phys., 24, 28-44, 1952.

Krimigis, S. M., and T. P. Armstrong, Two-component proton spectra in the inner Saturnian magnetosphere, Geophys. Res. Lett., 9, 1143-1146, 1982.

Krimigis, S. M., T. P. Armstrong, W. I. Axford, C. O. Bostrom, G. Gloeckler, E. P. Keath, L. J. Lanzerotti, J. F. Carbary, D. C. Hamilton, and E. C. Roelof, Low energy charged particles in Saturn's magnetosphere: Results from Voyager 1, Science, 212, 225-230, 1981.

Marouf, E. A., G. L. Tyler, and V. R. Eshleman, Theory of radio occultation by Saturn's rings, Icarus, 49, 161-193, 1982.

McDonald, F. B., A. W. Schardt, and J. H. Trainor, If you've seen one magnetosphere, you haven't seen them all: Energetic particle observations in the Saturn magnetosphere, J. Geophys. Res., 85, 5813-5830, 1980.

Nakada, M. P., High-energy electrons in the radiation belt, J. Geophys. Res., 68, 47-53, 1963.

Nakada, M. P., and G. D. Mead, Diffusion of protons in the outer radiation belt, J. Geophys. Res., 70, 4777-4791, 1965.

Paonessa, M., and A. F. Cheng, Limits on ion radial diffusion coefficients in Saturn's inner magnetosphere, J. Geophys. Res., 91, 1391-1396, 1986.

Scarf, F. L., D. A. Gurnett, and W. S. Kurth, Measurements of plasma wave spectra in Jupiter's magnetosphere, J. Geophys. Res., 86, 8181-8198, 1981.

Scarf, F. L., D. A. Gurnett, W. S. Kurth, and R. L. Poynter, Voyager plasma wave measurements at Saturn, J. Geophys. Res., 88, 8971-8984, 1983.
Schardt, A. W., and F. B. McDonald, The flux of energetic protons in Saturn's inner magnetosphere, $J$. Geophys. Res., 88, 8923-8935, 1983.

Schula, M., Approximate second invariant for a dipole field, J. Geophys. Res., 76, 3144-3148, 1971.

Schulz, M., and L. J. Lanzerotti, Particle Diffusion in the Radiation Belts, Springer-Verlag, New York, 1974.

Smith, B. A., The D and E rings of Saturn, in The Saturn System, NASA Conf. Publ. 2068, 105-111, 1978.

Smith, E. J., L. Davis, Jr., D. E. Jones, P. J. Coleman, Jr., D. S. Colburn, P. Dyal, and C. P. Sonett, Saturn's magnetosphere and its interaction with the solar wind, J. Geophys. Res., 85, 5655-5674, 1980.

Tabata, T., and R. Ito, A generalized empirical equation for the transmission coefficient of electrons, Nucl. Instrum. Methods, 127, 429-434, 1974.

Thomsen, M. F., and J. A. Van Allen, On the inference of properties of Saturn's ring E from energetic charged particle observations, Geophys. Res. Lett., 6, 893-896, 1979.

Van Allen, J. A., Findings on rings and inner satellites of Saturn by Pioneer 11, Icarus, 51, 509-527, 1982.

Van Allen, J. A., Absorption of energetic protons by Saturn's ring G, J. Geophys. Res., 88, 6911-6918, 1983.

Van Allen, J. A., Energetic particles in the inner magnetosphere of Saturn, in Saturn, edited by T. Gehrels and M. Matthews, pp. 281-317, University of Arizona Press, Tucson, Ariz., 1984.

Van Allen, J. A., and C. L. Grosskreutz, Relativistic electrons in Saturn's inner magnetosphere and an estimate of their synchrotron emission, J. Geophys. Res., 94, 87318738, 1989.

Van Allen, J. A., M. F. Thomsen, B. A. Randall, R. L. Rairden, and C. L. Grosskreutz, Saturn's magnetosphere, rings, and inner satellites, Science, 207, 415-421, 1980a.

Van Allen, J. A., B. A. Randall, and M. F. Thomsen, Sources and sinks of energetic electrons and protons in Saturn's magnetosphere, J. Geophys. Res., 85, 5679-5694, 1980b.

Van Allen, J. A., M. F. Thomsen, and B. A. Randall, The energetic charged particle absorption signature of Mimas, J. Geophys. Res., 85, 5709-5718, 1980c.

Vogt, R. E., D. L. Chenette, A. C. Cummings, T. L. Garrard, E. C. Stone, A. W. Schardt, J. H. Trainor, N. Lal, and F. B. McDonald, Energetic charged particles in Saturn's magnetosphere: Voyager 2 results, Science, 215, 577$582,1982$.

Zuyagaina, E. V., G. V. Pechernikova, and V. S. Safronou, Qualitative solutions of the coagulation equations with allowance for fragmentation, Sov. Astron., Engl. Transl., 17, 793-800, 1974.

B. A. Randall, Department of Physics and Astronomy, University of Iowa, Van Allen Hall, Iowa City, IA 522421479. (e-mail:SPAN:iowasp::randall)

(Received May 21, 1993; revised December 14, 1993; accepted January 20, 1994.) 
